\title{
The future of genomics in pathology
}

\author{
Dennis P. Wall ${ }^{1,2} *$ and Peter J. Tonellato ${ }^{1,2}$
}

\author{
Addresses: ${ }^{1}$ Department of Pathology, Beth Israel Deaconess Medical Center, 330 Brookline Avenue, Boston, MA 02215; ${ }^{2}$ Center for Biomedical \\ Informatics, Harvard Medical School, Boston, MA \\ *Corresponding author: Dennis P. Wall (dpwall@hms.harvard.edu) \\ Fl000 Medicine Reports 2012, 4:14 (doi:10.3410/M4-14) \\ This is an open-access article distributed under the terms of the Creative Commons Attribution-Non Commercial License \\ (http://creativecommons.org/licenses/by-nc/3.0/legalcode), which permits unrestricted use, distribution, and reproduction in any medium, \\ provided the original work is properly cited. You may not use this work for commercial purposes. \\ The electronic version of this article is the complete one and can be found at: http://f1000.com/reports/m/4/I4
}

\begin{abstract}
The recent advances in technology and the promise of cheap and fast whole genomic data offer the possibility to revolutionise the discipline of pathology. This should allow pathologists in the near future to diagnose disease rapidly and early to change its course, and to tailor treatment programs to the individual. This review outlines some of these technical advances and the changes needed to make this revolution a reality.
\end{abstract}

\section{Introduction}

Pathology is the branch of medicine that diagnoses the nature of much human disease and forms the basis for treatment. It is the division of the hospital where your blood samples, cultures, Pap smears, and biopsies are sent. Pathologists are experts at interpreting microscopic views of body tissues, and are the responsible authority on making diagnoses that lead to disease treatment and prevention. When your doctor tells you that you have elevated cholesterol and prescribes exercise and Lipitor, he or she is acting on the results of a test report from a pathologist. And, when your doctor tells you they are waiting for the test results, he or she is really waiting for a pathologist to provide a definitive interpretation and clinical description of one or more diagnostic tests and data conducted on the blood or other tissue you provided earlier. In short, pathology provides the fundamental lab tests, data and clinical interpretation (to the tune of 7.1 billion laboratory tests in 2009) that form the core of a patient's medical record and the data that drives healthcare.

While the field of pathology dates back to the Greek physician Hippocrates, pathology's impact on health has relied heavily on a steady stream of technological advances. The invention and enhancement of the light microscope [1] enabled the rapid evolution in our understanding of infectious diseases and concomitantly launched the field of modern pathology through the efforts of Rudolph Virchow [2]. The invention of the electron microscope in the 20th century enabled major advances in our ability to differentiate states and stages of diseases. The invention of nucleic acid-based diagnostics represented another key innovation for the field of pathology, and paved the way to more complex multiplex testing with dozens of tests possible from a single blood sample. In a similar way, digital imaging, facilitated by advancements in both emission energy detection and computer technology, likewise has generated a new platform for pathology to conduct high-resolution diagnostic testing, and thereby provides more detailed quantification of the diagnoses used in the clinical discrimination of disease and corresponding insight and guidance into more individualized disease treatments.

Nevertheless, the rate of uptake of new technologies by the field of pathology has been slow. The punctuated jumps forward in our understanding of infectious disease and ability to conduct diagnostics with the compound microscope came nearly 150 years after its invention. Today, nearly 80 years after the invention of the electron microscope, disagreement remains about its full value in diagnostic practice. Digital imaging took nearly ten years to penetrate into standard practices of medical pathology, despite being established in virtually all other branches of the hospital, and polymerase chain reaction (PCR) 
technologies took approximately 5 years to take hold, again despite widespread adoption in medical research. In all cases, by the time these critical tools percolated into the standard practice of hospital pathology they were no longer new, but well established, and in some cases already being replaced by alternative strategies and competing technologies.

There is no doubt that the stereotype of the pathologist wearing tweed, studying slides under a microscope alongside a dead body persists in part because of this seeming reluctance to adopt new technologies. At the same time, there is no doubt that pathology has remained, and likely will remain, the backbone of the quantification and laboratory testing used throughout healthcare. Regardless of the historical delays to assimilate new technologies into pathology best practice and in spite of the typical barriers to any new technology adoption, a "game-changing" technological innovation has emerged that the field of pathology cannot afford to ignore.

The innovation is next generation sequencing. Unlike other inventions that have sharpened the clinical impact of pathology, genomic sequencing technologies are undergoing regular upheavals at unprecedented speeds. Parallel developments in ligation-based methods, pyrosequencing, polony-based approaches, and singlemolecule sequencing have created a fiercely competitive marketplace that is challenging historical leaders in the field, including array-centric companies, while simultaneously bankrupting early pioneers of newer techniques. In this competitive landscape, quality and speed are both rocketing upward. The fastest technologies can sequence an entire human genome, 3 billion bases, in hours, and corresponding costs have dropped from $\$ 30,000$ to $\$ 4,000$ within about a year. And while there remains some variation in quality, speed, and cost, that variation is quickly evaporating through market demand. The thousand dollar genome will soon arrive [3].

\section{Genomic pathology}

The field of pathology cannot afford to ignore the next generation sequencing innovation because, in the hands of medical pathologists, it has the potential to reshape the fabric of healthcare and make personalized medicine a ubiquitous reality. Another reason is that unlike all others that have advanced the impact of pathology on healthcare, the practice of next generation whole genome analysis can, should, and will replace many current standard pathology practices of diagnosis and prognosis on which proper therapy and disease management rely today, the same best practice standards of pathology that drive considerable proportions of the clinical decision-making process $[4,5]$. Pathologists are the direct link between analysis and interpretation of medical data and care delivery: they order, conduct, and interpret the results of hundreds of localized tests to arrive at the medical conclusions that enable proper care. A large percentage of these tests are commoditized molecular diagnostics, outsourced to the appropriate provider. While every test represents an innovation in healthcare, it is the pathologist's interpretation of the test results that actually leads to improvements in health, and next generation sequencing data is no different. Just like standard molecular diagnostics, one can order the genome and/or transcriptome for the patient, but it is the proper clinical interpretation that will add the value for healthcare. It is difficult to imagine next generation genomics having any tangible impact on health if fractionated throughout multiple branches of the hospital. However, for pathology to succeed at translating this innovation into actionable health information, pathology departments must shed their stodgy stereotype and rise to the challenge of not only reacting in an appropriate timeframe to the innovation of next generation genomics and all that comes with it but also addressing and resolving the challenges presented by key barriers to entry.

Whole genomic data should become a vital component of the healthcare enterprise, and when one considers the historical role pathology has played in this enterprise, it is clear that pathology is the most logical home for whole genome analysis-based clinical decision support.

\section{Personalized care through genomic pathology: steps and barriers}

We have begun to establish a national agenda for the future of pathology in personalized medicine [6], highlighting key areas that must be addressed in order for pathology to play a leading role in the clinical application of whole genome analysis. These are (1) defining clinical actionability, (2) designing a clinical interpretation system to generate medical impact reports from next generation sequencing data and whole genome analysis, and (3) training a new breed of genomic pathologist through novel, national education programs.

\section{Defining clinical actionability}

Guidelines and recommendations have been developed [7-9] that will help guide the process of developing a nationally agreed upon set of criteria that must be met in order for whole genomic analysis to penetrate effectively into the clinic. These recommendations and guidelines need re-evaluation within the context of recent technological advances. Taking a step forward, we propose that the definition of clinical actionability should be focused on genomic analysis, which qualifies for medical insurance cover in countries like the USA and is provided 
by state-funded health services in countries that have them. Such analysis is geared towards specific clinical outcomes minimally including treatment and prognosis risk assessment. With this functional definition, a variant is clinically actionable if, for example, the variant is used to determine whether a patient with cancer will respond to erlotinib, sunitinib, neither, and, if neither, whether a clinical trial is underway and enrolling new candidates with that variant for experimental treatment. Proof of concept exists that an individual's genome can inform care [10], and additional evidence is mounting, but now is the time to settle on the minimum criteria for clinically actionable genomic variants. While whole genome analysis will, at least for some period of time, generate ancillary findings, the field of pathology has a rich history of handling ancillary data within clinical contexts. The autoanalyzer technology developed by Skeggs in the 1950 s was yet another key innovation that enabled a leap forward in pathology-driven precision medicine [11], enabling dozens of tests to be conducted simultaneously with high accuracy and speed. However, while all tests were done simultaneously, not all were relevant to the clinical context under study. These ancillary findings are of the same nature, though of smaller dimensionality, as whole genome analysis data. In this context, pathology is positioned to play the key role in the implementation of whole genome analysis as a clinical test, with the understanding that genomic testing is fundamentally no different from other types of laboratory testing. We take the stance that "clinical grade genomics" and "clinically actionable variation" must fall under the same type of regulatory oversight as other clinical testing and therefore that the College of American Pathologists and other international/national pathology organizations should seize the initiative and develop global/international standards and regulations governing genome testing. Having such regulation in place will ensure repeatability and measurable quality, but will also facilitate the dialogue between medical insurance and government agencies on the one hand and medical practices on the other and enable faster resolution of reimbursement funding questions.

\section{Building a clinical variant interpretation system}

Virtually every aspect of whole genome analysis can be commoditized and reliably outsourced, save clinical grade interpretation. This last step is the most crucial and involves a novel close coupling between clinicians and biomedical informatics specialists. Proper clinical interpretation requires integration of numerous layers of information that pathologists are trained in, but the analysis of whole genomics data adds a volume of scale and complexity that requires a new kind of sophistication and knowledge. Scaling up from standard molecular diagnostics to genomics in the pathology lab is contingent on having an interpretation system in place that enables rapid assessment within a clinical time window.

The natural starting point will be to determine and validate how, when and where whole genome analysis can replace the standard set of molecular diagnostic tests being conducted today. Based on an analysis of the Association for Molecular Pathology's solid tumor test directory [12], over 600 tests are in use for analysis and clinical interpretation of solid tumors for more than 90 conditions. By mapping these into a network of test types (e.g. mutational analysis or amplification-based test) and cancer types, we calculate that $85 \%$ will be immediately replaceable with whole genome analysis for multiplex clinical annotation and medical impact assessment (Figure 1).

Pathology, by enlisting new professionals in genomic medicine, needs to gain control of the effort to establish a certified, clinical grade variant database. Current sequence variant databases have been built through an ad hoc process designed to support research activities. They fall short of what is needed for delivery of accurate, safe and effective patient care. Clinical laboratory testing using human genome sequence data requires the creation, ongoing support, and national regulatory oversight of a clinical grade database. With this in place, we will be able to establish standard best practices quickly and propagate new genomic discoveries nationwide with a simple click of a button. These discoveries can then be integrated with other critical pieces of medical record data to inform decisions within a clinical timeframe. Such data should include standard histopathology, but should also soon include prediction data from digital slide scanning and image analysis technologies, a field that is developing at a rapid pace and that too holds tremendous potential for rapid, fine grained and clinically accurate disease characterization [13].

\section{Training the future "genomic medicine" pathologists}

We have to train our future doctors to understand the whole genomics analysis pipeline and to be able to interpret medical impact reports that contain genomically informed diagnoses. Pathology programs have to embrace the innovation of next generation genomics by establishing residency training programs that provide all the skills necessary to interpret and act on whole genomic data. This is already happening now [14]. In the US, a national committee has been formed, including members of the Pathology Program Directors (PRODS) and other key stakeholders, to disseminate model curricula and support their widespread implementation. We are moving fast toward definitions of the core competencies in genomics and personalized medicine, and it is likely that, within 


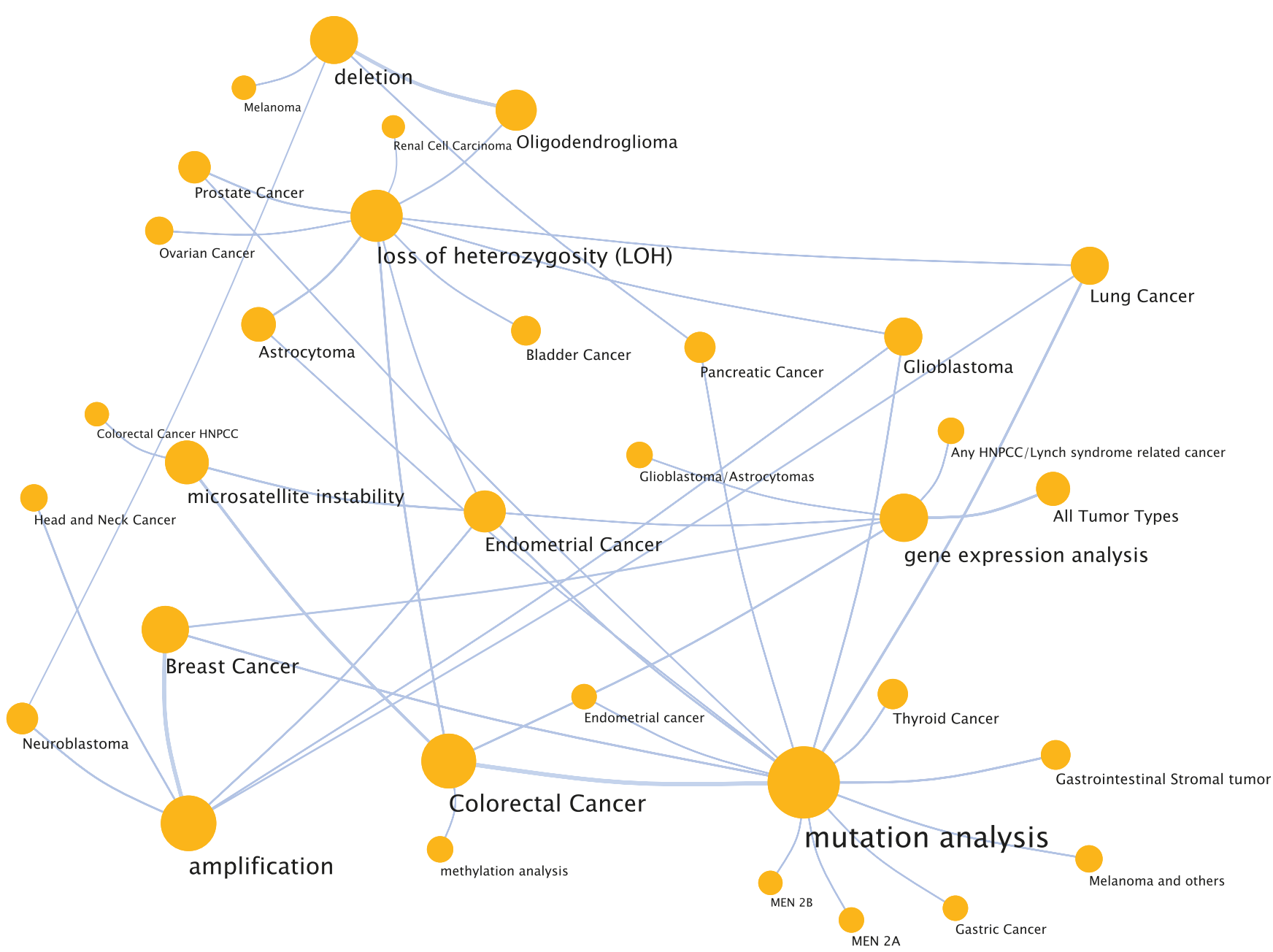

A network view of a sample of tests and diseases found in the Association for Molecular Pathology's solid tumor test directory [12]. A total of 45 nodes and 96 edges are depicted in the figure. The nodes are the most common tests and diseases in the directory and edges are drawn from tests to disease if the test is used commonly for disease characterization. The extent of genetic testing suggests that next generation sequencing and whole genomic analysis approaches have the potential to replace $85 \%$ of the tests found in this directory, greatly simplifying the network and having the potential to streamline the industry.

two years, it will be required that all residents in pathology demonstrate proficiency in these areas.

\section{Conclusions}

The promise of whole genomic data is within reach. We will be able to diagnose rapidly and early to change the course of disease, to prevent adverse drug combinations and promote beneficial ones, to prevent inappropriate dosing, and to tailor treatment programs to the individual. This will dramatically improve health while staving off massive failures in the pharmaceutical industry such as rofecoxib and celecoxib [15]. However, this transformation of "personalized genomics" from party hype into clinical action requires disruptive computational innovation at the heart of the health care enterprise. This is pathology's time to seize the opportunity in pace and be in tune with the technological innovations of modern human genomics. Getting there will require a national revamp of hospital pathology to ensure that modern genomic practices are deeply integrated with and regulated by the same standards as any other clinical test in patient care. In short, we must embed high-powered computational analysis and clinical assessment of human genomes directly into the pipeline of standard care testing 
and delivery. This may be the only way to guarantee that the "thousand dollar genome" achieves its potential to make personalized medicine a widespread reality.

\section{Abbreviation}

PRODS, Pathology Program Directors; PCR, Polymerase chain reaction

\section{Competing interests}

The authors declare that they have no competing interests.

\section{References}

I. Kohler A: Gedanken zu einem neuen Beleuchtungsverfahren für mikrophotographische Zwecke. Zeitschrift für wissenschaftliche Mikroskopie 1893.

2. Ackerknecht EH: Rudolf Virchow: Doctor, statesman, anthropologist. Madison: University of Wisconsin; 1953.

3. Davies K: The $\$ 1000$ Genome. New York: Free Press; 2010.

4. Forsman RW: Why is the laboratory an afterthought for managed care organizations? Clin Chem 1996, 42:8I3-6.

5. The Lewin Group: The Value of Diagnostics Innovation, Adoption and Diffusion into Health Care. The Lewin Group Inc; 2005. [http://www. socalbio.org/pdfs/thevalueofdiagnostics.pdf]

6. Tonellato PJ, Crawford JM, Boguski MS, Saffitz JE: A national agenda for the future of pathology in personalized medicine: report of the proceedings of a meeting at the Banbury Conference Center on genome-era pathology, precision diagnostics, and preemptive care: a stakeholder summit. Am J Clin Pathol 201 I, 135:668-72.

7. Richards CS, Bale S, Bellissimo DB, Das S, Grody WW, Hegde MR, Lyon E, Ward BE: ACMG recommendations for standards for interpretation and reporting of sequence variations: Revisions 2007. Genet Med 2008, I 0:294-300.

FI000 Factor 6

Dennis Wall 20 Jun 2012

8. Das S, Bale SJ, Ledbetter DH: Molecular genetic testing for ultra rare diseases: models for translation from the research laboratory to the CLIA-certified diagnostic laboratory. Genet Med 2008, 1 0:332-6.

9. Maddalena A, Bale S, Das S, Grody W, Richards S: Technical standards and guidelines: molecular genetic testing for ultrarare disorders. Genet Med 2005, 7:57I-83.

10. Jones SJ, Laskin J, Li YY, Griffith OL, An J, Bilenky M, Butterfield YS, Cezard T, Chuah E, Corbett R, Fejes AP, Griffith M, Yee J, Martin M, Mayo M, Melnyk N, Morin RD, Pugh TJ, Severson T, Shah SP, Sutcliffe M, Tam A, Terry J, Thiessen N, Thomson T, Varhol R, Zeng T, Zhao Y, Moore RA, Huntsman DG, et al.: Evolution of an adenocarcinoma in response to selection by targeted kinase inhibitors. Genome Biol 2010, I I:R82.

II. Skeggs LT: Persistence... and prayer: from the artificial kidney to the AutoAnalyzer. Clin Chem 2000, 46:1425-36.

12. AMP Test directories for infectious diseases, solid tumors and hematopathology. [http://www.amptestdirectory.org/STTestDirectory.htm]

13. Beck AH, Sangoi AR, Leung S, Marinelli RJ, Nielsen TO, van de Vijver MJ, West RB, van de Rijn M, Koller D: Systematic analysis of breast cancer morphology uncovers stromal features associated with survival. Sci Transl Med 20I I, 3:I08ra I I3.

FI000 Factor 6

Peter Tonellato 20 Jun 2012

14. Haspel RL, Arnaout R, Briere L, Kantarci S, Marchand K, Tonellato P, Connolly J, Boguski MS, Saffitz JE: A call to action: training pathology residents in genomics and personalized medicine. Am J Clin Pathol 2010, 133:832-4.

I5. Brownstein JS, Sordo M, Kohane IS, MandI KD: The tell-tale heart: population-based surveillance reveals an association of rofecoxib and celecoxib with myocardial infarction. PLOS ONE 2007, 2:e840. 